\title{
Correction to: Risk factors for post-nephrectomy hypotension in pediatric patients
}

\author{
Kentaro Nishi ${ }^{1}$ - Koichi Kamei ${ }^{1}$ (i) $\cdot$ Masao Ogura $^{1} \cdot$ Mai Sato $^{1} \cdot$ Sho Ishiwa ${ }^{1,2} \cdot$ Yoko Shioda $^{3} \cdot$ Chikako Kiyotani $^{3}$. \\ Kimikazu Matsumoto $^{3} \cdot$ Kandai Nozu $^{4} \cdot$ Kenji Ishikura $^{1,5}$. Shuichi Ito ${ }^{1,6}$
}

Published online: 12 July 2021

(C) IPNA 2021

\section{Correction to: Pediatric Nephrology} https://doi.org/10.1007/s00467-021-05115-7

The original version of this article, published on 14 May 2021, unfortunately, contained a mistake.

In Table 4 of this article, the data in column "P value," row "Age at nephrectomy (year)," contained a typesetting mistake. The table should have appeared as shown below.

The publisher apologizes for this mistake.

The original article has been corrected.

Publisher's note Springer Nature remains neutral with regard to jurisdictional claims in published maps and institutional affiliations.

The online version of the original article can be found at https://doi.org/ 10.1007/s00467-021-05115-7

Koichi Kamei

kamei-k@ncchd.go.jp

1 Division of Nephrology and Rheumatology, National Center for Child Health and Development, 2-10-1, Okura, Setagaya-ku, Tokyo 157-8535, Japan

2 Department of Pediatric Nephrology, Tokyo Women's Medical University, Tokyo, Japan

3 Children's Cancer Center, National Center for Child Health and Development, Tokyo, Japan

4 Department of Pediatrics, Kobe University Graduate School of Medicine, Kobe, Hyogo, Japan

5 Department of Pediatrics, Kitasato University School of Medicine, Sagamihara, Kanagawa, Japan

6 Department of Pediatrics, Yokohama City University Graduate School of Medicine, Yokohama, Kanagawa, Japan 
Table 4 Comparison between patients with and without post-nephrectomy hypotension

\begin{tabular}{|c|c|c|c|c|}
\hline & Hypotension $(\mathrm{n}=11)$ & No hypotension $(\mathrm{n}=44)$ & $P$ value \\
\hline \multicolumn{2}{|c|}{ Male sex } & $9(81)$ & $20(45)$ & 0.04 \\
\hline \multicolumn{5}{|c|}{ Primary disease } \\
\hline \multicolumn{2}{|c|}{ Isolated renal tumor } & $2(18)$ & $22(50)$ & \\
\hline \multicolumn{2}{|c|}{ Wilms tumor/RTK/CCSK } & $1 / 0 / 1$ & $19 / 1 / 2$ & \\
\hline \multicolumn{2}{|c|}{ Neuroblastoma } & $1(9)$ & $9(20)$ & \\
\hline \multicolumn{2}{|l|}{ CNS } & $3(27)$ & $8(18)$ & \\
\hline \multicolumn{2}{|c|}{ NPHS1/NPHS2 mutations } & $3 / 0$ & $7 / 1$ & \\
\hline \multicolumn{2}{|c|}{ WT1 nephropathy } & $3(27)$ & $5(11)$ & \\
\hline \multicolumn{2}{|c|}{ DDS/WAGR syndrome } & $3 / 0$ & $4 / 1$ & \\
\hline \multicolumn{2}{|c|}{ ARPKD } & $2(18)$ & $0(0)$ & \\
\hline \multicolumn{2}{|c|}{ Renal diseases } & $8(72)$ & $13(29)$ & 0.01 \\
\hline \multicolumn{2}{|c|}{ Age at nephrectomy (year) } & $1.9(0.03-15.9)$ & $2.1(0.16-14.6)$ & 0.49 \\
\hline \multicolumn{2}{|c|}{ Resection of both kidneys } & $5(45)$ & $4(9)$ & $<0.001$ \\
\hline \multicolumn{5}{|c|}{ Renal function at nephrectomy } \\
\hline \multicolumn{2}{|c|}{ Normal } & $3(27)$ & $23(52)$ & \\
\hline \multirow[t]{5}{*}{ CKD } & Stage 1 eGFR >90 (mL/min/1.73 m²) & 0 & $3(7)$ & \\
\hline & Stage 2 eGFR $60-90\left(\mathrm{~mL} / \mathrm{min} / 1.73 \mathrm{~m}^{2}\right)$ & $1(9)$ & $8(18)$ & \\
\hline & Stage 3 eGFR $30-60\left(\mathrm{~mL} / \mathrm{min} / 1.73 \mathrm{~m}^{2}\right)$ & 0 & $3(7)$ & \\
\hline & Stage 4 eGFR $15-30\left(\mathrm{~mL} / \mathrm{min} / 1.73 \mathrm{~m}^{2}\right)$ & 0 & $1(2)$ & \\
\hline & Stage 5 eGFR $<15\left(\mathrm{~mL} / \mathrm{min} / 1.73 \mathrm{~m}^{2}\right)$ & 0 & $1(2)$ & \\
\hline \multicolumn{2}{|c|}{ On dialysis } & $7(64)$ & $5(11)$ & \\
\hline \multicolumn{2}{|c|}{$\mathrm{PD} / \mathrm{HD}$} & $4 / 3$ & $5 / 0$ & \\
\hline \multicolumn{2}{|c|}{ eGFR at nephrectomy $\left(\mathrm{mL} / \mathrm{min} / 1.73 \mathrm{~m}^{2}\right)$} & $97.6(5.9-128.1)$ & $100.3(5.6-155.7)$ & 0.04 \\
\hline \multicolumn{2}{|c|}{ Serum albumin $(g / d L)$} & $3.1(1.6-4.6)$ & $2.8(0.8-3.8)$ & 0.43 \\
\hline \multicolumn{5}{|c|}{ Cardiac function } \\
\hline \multicolumn{2}{|c|}{ LVEF $(\%)$} & $69(52-82)$ & $70.5(52-81)$ & 0.36 \\
\hline \multicolumn{2}{|c|}{ IVSd (SD) } & $2.9(-0.1$ to 5.4$)$ & $1.45(-1.6-7.8)$ & 0.07 \\
\hline \multicolumn{2}{|c|}{ LVPWd (SD) } & $3.2(-1.8$ to 7.8$)$ & $0.5(-1.7-4.6)$ & 0.008 \\
\hline \multicolumn{2}{|l|}{$\mathrm{E} / \mathrm{E}^{\prime}$} & $10.6(2.8-11.9)$ & $9.9(4.8-16)$ & 0.71 \\
\hline \multicolumn{2}{|c|}{ Hypertension before nephrectomy } & $8(72)$ & $7(15)$ & $<0.001$ \\
\hline \multicolumn{2}{|c|}{ Use of anti-hypertensives before nephrectomy } & $7(63)$ & $12(27)$ & 0.03 \\
\hline \multicolumn{2}{|c|}{ Hyperreninemia before nephrectomy } & $5^{\mathrm{a}}(100)$ & $7^{\mathrm{b}}(41)$ & 0.03 \\
\hline \multicolumn{2}{|c|}{ Hyperaldosteronism before nephrectomy } & $5^{\mathrm{a}}(100)$ & $2^{\mathrm{c}}(15)$ & 0.002 \\
\hline
\end{tabular}

Data are expressed as numbers (\%) ormedians (min-max). eGFR, estimated glomerular filtration rate; $P D$, peritoneal dialysis; $H D$, hemodialysis; $L V E F$, left ventricular ejection fraction; $I V S d$, interventricular septal thickness at diastole; $L V P W d$, left ventricular posterior wall thickness in diastole; E/e ratio, mitral inflow/annular ratio. ${ }^{\mathrm{a}}$ Measured in 5 cases; ${ }^{\mathrm{b}}$ Measured in 17 cases; ${ }^{\mathrm{c}}$ Measured in 13 case 